\title{
DESENVOLVIMENTO E AVALIAÇÃO SENSORIAL DE CONFEITO DE AVEIA COM ALFARROBA ISENTO DE LACTOSE
}

\section{DEVELOPMENT AND SENSORY EVALUATION OF A LACTOSE FREE OAT CEREAL BALL WITH CAROB}

\author{
Aline Veroneze de Mello ${ }^{1}$ \\ Walleska Luctke Facincani Villarin ${ }^{1}$ \\ Mônica Glória Neumann Spinelli² \\ Andrea Carvalheiro Guerra Matias ${ }^{2}$
}

Resumo: O objetivo do presente estudo foi avaliar a aceitabilidade de um confeito de aveia com cobertura de alfarroba isento de lactose. Para tanto, o produto foi submetido a um teste de aceitabilidade para os atributos aparência, odor, textura, sabor e aceitação global, bem como avaliação de intenção de compra. O produto apresentou boa aceitação com notas médias acima de 7 (gostei regularmente), e Índice de aceitabilidade acima de $80 \%$ para todos os atributos. Quanto à intenção de compra, 84\% dos degustadores "comprariam" ou, "definitivamente", comprariam o produto. O confeito apresenta 1,6g de fibra alimentar por porção, recebendo a informação nutricional complementar de fonte. O estudo atingiu seus objetivos obtendo um produto isento de lactose, fonte de fibras e com boa aceitação sensorial.

Palavras-chave: alfarroba; aveia; análise sensorial; novos produtos.

\begin{abstract}
The aim of this study was to evaluate the acceptability of a lactose free cereal ball prepared with oatmeal and topped with carob. A sensorial acceptability test was conducted for attributes of appearance, odor, texture, flavor, and overall acceptability, as well as to evaluate intention to purchase. The product was well accept, with average scores above 7 (regularly enjoyed), and acceptability index above $80 \%$ for all attributes. For the intention to purchase, $84 \%$ of the panel option to "buy" or "definitely buy" the product. The cereal ball has $1.6 \mathrm{~g}$ of dietary fiber in the portion, and it is a source of dietary fiber. The study achieved its objectives obtaining a lactose free product, which is source of fiber and had good acceptability.
\end{abstract}

Keywords: carob; oat; sensory evaluation; new products.

\footnotetext{
1 Graduanda em Nutrição - Universidade Presbiteriana Mackenzie - UPM. E-mails: alinevm3@hotmail.com; walleskao@hotmail.com.

2 Doutora em Saúde Pública - Universidade de São Paulo - USP e Docente da UPM. E-mails: monicaspi404@gmail.com; acgmatias@gmail.com.
} 


\section{INTRODUÇÃO}

O desenvolvimento de novos produtos alimentícios torna-se cada vez mais desafiador à medida que procura atender à demanda dos consumidores por produtos que, simultaneamente, sejam saudáveis e atrativos (BURITI; SAAD, 2008). Os alimentos para fins específicos são especialmente formulados ou processados de forma que sejam introduzidas modificações no conteúdo de nutrientes, atendendo às necessidades de pessoas que possuem problemas alimentares específicos como intolerância à lactose, doença celíaca, diabetes, alergias, fenilcetonúria, entre outros (BRASIL, 1998; ABIAD, 2011).

As barras de careais são produtos que atendem vários segmentos de consumidores, uma vez que são consideradas uma alternativa saudável e prática para lanches consumidos por jovens e adultos. Essa tendência foi percebida pelo setor de alimentos, que hoje já desenvolveu uma grande variedade de sabores e finalidades (BOWER; WHITTEN, 2000; PALAZZOLO, 2003).

Em geral, as barras de cereal são constituídas de ingredientes secos, sendo os cereais em maior proporção, e agentes ligantes (uma mistura composta por açúcares e gorduras podendo conter aromatizantes). Esses confeitos são geralmente embalados e comercializados em porções individuais de 25 a 30 gramas (MURPHY, 1995).

A intolerância à lactose caracteriza-se pela ausência ou deficiência da enzima lactase, responsável pela sua digestão. A lactose é um dissacarídeo formado por glicose e galactose presentes no leite e seus derivados e, pela ausência da sua absorção no intestino delgado, é fermentada pelas bactérias intestinais, gerando ácido láctico, metano e gás hidrogênio, que, em conjunto, geram desconfortos gástricos caracterizados por cólicas intestinais, flatulência e diarreia. Dentre as formas de tratamento da intolerância à lactose, destaca-se evitar o consumo de produtos que contenham lactose ou consumi-los em menor quantidade (FRYE, 2002; MATTAR; MAZO, 2010). Dentro desse contexto, é necessário que o mercado tenha a preocupação de disponibilizar aos consumidores alimentos que sejam livres de lactose, para que esses grupos de pessoas tenham acesso a alimentos saborosos e tenham garantido o seu bem estar (FLORES, 2010).

Alfarroba (Ceratonia siliqua L.) é o fruto da alfarrobeira, uma árvore selvagem, nativa da costa do mediterrâneo. É uma vagem comestível, semelhante ao feijão, com uma coloração marrom escuro e com sabor adocicado. É uma leguminosa muito utilizada pela indústria de alimentos para a fabricação de gomas e espessantes, e o pó derivado da polpa da vagem torrada e moída pode ser utilizado para substituir o cacau (OZCAN, 2007).

Este trabalho teve como intuito elaborar e avaliar, sensorialmente, um confeito inspirado nas barras de cereal, no formato de esfera, isento de lactose à base de cereais com cobertura de alfarroba, com vistas a atender a demanda por produtos para indivíduos intolerantes ao leite, bem como prover uma opção de produto prático e saudável. 


\section{MATERIAL E MÉTODOS}

O desenvolvimento do produto e a análise sensorial foram realizados na Cozinha Experimental de uma universidade privada do município de São Paulo.

\subsection{Elaboração do produto}

Para o preparo, os ingredientes (Quadro 1), foram pesados, com auxílio de balança da marca Filizola®, com capacidade de $15 \mathrm{~kg}$. Posteriormente, foi elaborada uma calda com: água, leite em pó de soja, lecitina de soja, óleo de canola, maltodextrina e xarope de glucose (nessa ordem). Depois que a calda atingiu a temperatura de $110^{\circ} \mathrm{C}$ ("ponto de bala mole"), adicionaram-se os flocos finos de aveia, os flocos de arroz e a castanha do Pará (previamente misturados).

O produto foi moldado manualmente com a massa fria no formato de esferas de, aproximadamente, $2 \mathrm{~cm}$ de diâmetro, sendo acondicionadas sob refrigeração $\left(4^{\circ} \mathrm{C}\right)$ até 0 recebimento da cobertura de alfarroba. Para a cobertura, a alfarroba em barra foi derretida sob calor de micro-ondas. Depois de banhadas com a cobertura, as esferas de cereal foram novamente acondicionadas sob refrigeração.

A formulação do produto é apresentada no Quadro 1.

\section{Quadro 1 - Formulação do cereal de aveia com alfarroba}

\begin{tabular}{|l|r|}
\hline \multicolumn{1}{|c|}{ Ingredientes } & $\%$ \\
\hline Xarope de glucose & 33,2 \\
\hline Flocos finos de aveia & 18,9 \\
\hline Alfarroba em barra & 15,8 \\
\hline Maltodextrina & 9,5 \\
\hline Flocos de arroz & 9,5 \\
\hline Castanha do Pará & 6,0 \\
\hline Leite em pó de soja & 3,3 \\
\hline Óleo de canola & 3,3 \\
\hline Lecitina de soja & 0,5 \\
\hline
\end{tabular}

\subsection{Análise Sensorial}

Para verificar a aceitação do produto, foi realizada análise sensorial com painel de 54 provadores não treinados, formado por alunos e funcionários da universidade. A pesquisa atendeu aos princípios éticos da Resolução do CNS 196/96 e foi aprovada pelo CIEP no N001/02/11.

Foram considerados como critérios de exclusão, para participação da análise sensorial, alergia ou aversão a algum componente da fórmula, estar gripado, ou ter fumado na última hora anterior ao teste.

A degustação das amostras foi realizada em cabines individuais, e as amostras servidas em recipientes brancos, limpos, inodoros e descartáveis. 
Foi aplicado um teste afetivo de aceitabilidade com escala hedônica de 9 pontos, ancorada nos extremos "gostei extremamente" e "desgostei extremamente" (STONE; SIDEL, 1993). Os confeitos de cereal foram avaliados quanto aos atributos sabor, odor, textura, aparência e aceitação global. Também foi aplicado um teste de intenção de compra de 5 pontos, ancorado nos extremos "definitivamente compraria" e "definitivamente não compraria".

O Índice de Aceitabilidade (IA) foi calculado considerando a nota máxima alcançada como $100 \%$ e a pontuação média, em \%. Um produto bem aceito sensoriamente é aquele cujo valor de Al>70\% (TEIXEIRA; MEINERT; BARBETTA, 1987).

\subsection{Informação nutricional}

O valor nutricional do confeito de aveia com cobertura de alfarroba foi comparado a três produtos similares encontrados no comércio varejista: barras de cereais de aveia com cobertura de chocolate e dois confeitos de cereal (cereal ball) com cobertura sabor chocolate.

Os cálculos nutricionais do produto foram realizados com base na Tabela TACO (UNICAMP, 2011) e, na ausência de algum ingrediente da fórmula, foram utilizadas as informações disponíveis no rótulo do fabricante. Para os produtos comerciais, utilizaram-se as informações nutricionais declaradas no rótulo.

\section{RESULTADOS E DISCUSSÃO}

Do total de participantes, $87 \%$ eram do sexo feminino e 13\%, masculino, com idades entre 17 e 48 anos. Quanto à escolaridade, 57\% possuíam terceiro grau completo e 43\%, segundo grau completo.

Conforme apresentado na Tabela 1 e Figura 1, o confeito de cereal com alfarroba foi bem aceito pelo painel, visto que as médias para todos os atributos apresentaram valores acima de 7 (gostei moderadamente), com moda 8 (gostei muito) para os atributos odor, sabor, textura e aceitação global.

Tabela 1 - Estatística descritiva dos valores hedônicos do teste de aceitabilidade. São Paulo, 2012

\begin{tabular}{lccccc}
\hline \multicolumn{1}{c}{ Atributo } & Média & $\begin{array}{c}\text { Desvio } \\
\text { padrão }\end{array}$ & Moda & $\begin{array}{c}\text { Valor } \\
\text { mínimo }\end{array}$ & $\begin{array}{c}\text { Valor } \\
\text { máximo }\end{array}$ \\
\hline Aparência & 7,2 & 1,3 & 7 & 4 & 9 \\
Odor & 7,3 & 1,3 & 8 & 4 & 9 \\
Sabor & 7,5 & 1,3 & 8 & 4 & 9 \\
Textura & 7,7 & 1,1 & 8 & 4 & 9 \\
Aceitação global & 7,7 & 0,9 & 8 & 5 & 9 \\
\hline
\end{tabular}

Pela análise dos histogramas de aceitação por atributo (Figura 1), pode-se observar excelente aprovação do produto, visto que, para soma em porcentagem das notas 9 (gostei muitíssimo), 8 
(gostei muito), e 7 (gostei moderadamente), temos 93\% para sabor, 89\% para aceitação global, $83 \%$ para textura, $78 \%$ para odor, e $72 \%$ para aparência.

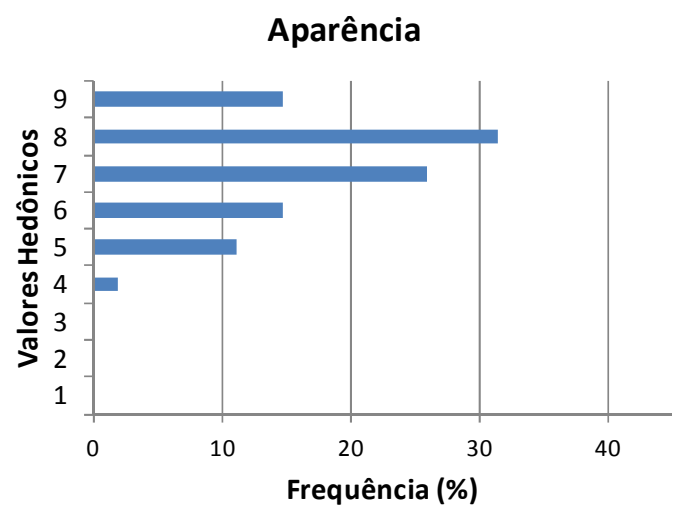

Textura

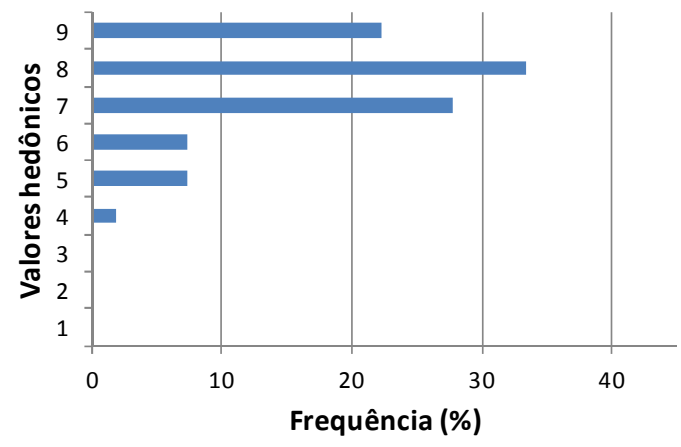

Odor

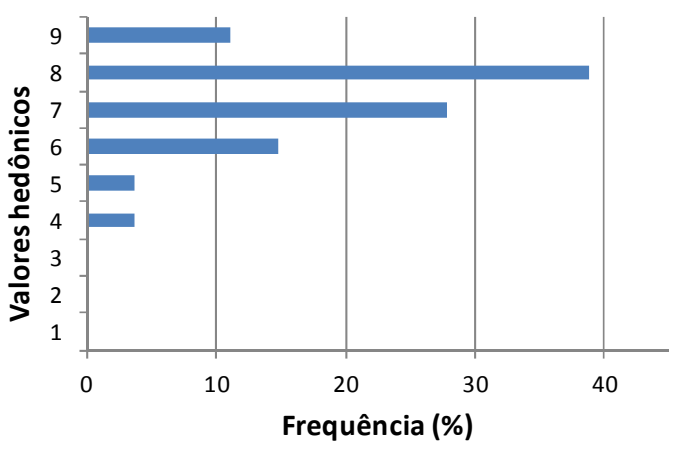

Sabor

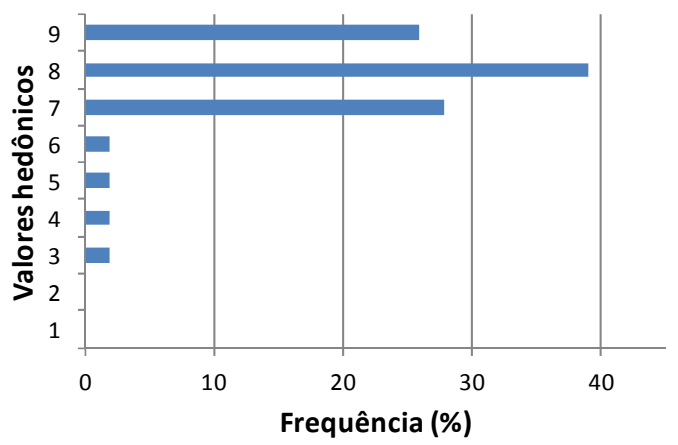

Aceitação Global

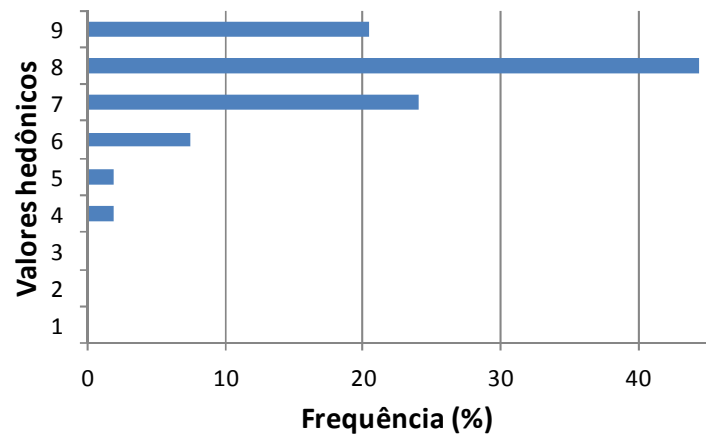

Figura 1 - Histogramas das notas do teste de aceitabilidade para os atributos aparência, odor, sabor, textura e aceitação global.

Esse resultado é corroborado pelos dados do Índice de Aceitabilidade, apresentados na Figura 2, na qual todos os atributos apresentaram valores acima de $70 \%$, o que indica boa aceitabilidade (TEIXEIRA; MEINERT; BARBETTA, 1987). 


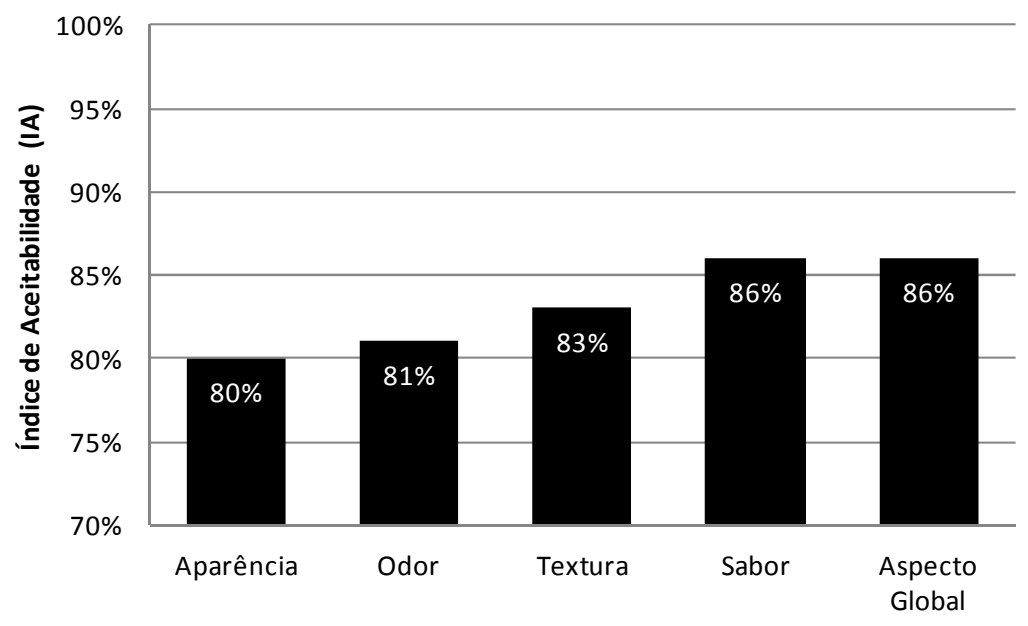

Figura 2 - Distribuição dos Índices de Aceitabilidade segundo atributo. São Paulo, 2012.

A boa aceitação do produto também é observada por intermédio dos dados de Intenção de compra, por meio dos quais a maioria dos degustadores declarou que "provavelmente compraria" ou "definitivamente compraria" o produto, representando $83,4 \%$ do total (Figura 3).

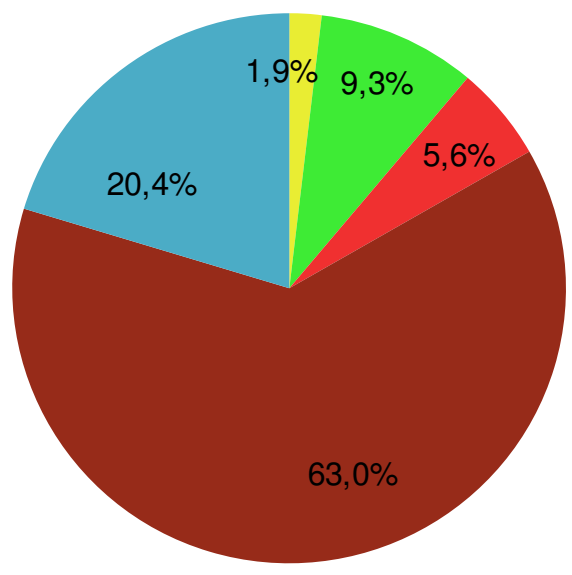

Definitivamente não compraria

Provavelmente não compraria

Não sei

Provavelmente compraria

Definitivamente compraria

Figura 3 - Distribuição em porcentagem da Intenção de compra. São Paulo, 2012.

No Quadro 2 são apresentados os valores nutricionais do confeito de aveia com alfarroba e três produtos similares comercializados no comércio varejista, sendo uma barra de cereal e dois confeitos (no formato de esfera) com cobertura de chocolate. 
Quadro 2 - Informação nutricional do confeito de cereal com alfarroba e produtos comerciais similares com chocolate. São Paulo, 2013

\begin{tabular}{|l|c|c|c|c|}
\hline \multicolumn{5}{|c|}{$\begin{array}{c}\text { INFORMAÇÃO NUTRICIONAL } \\
\text { PORÇO (30g) }\end{array}$} \\
\hline & $\begin{array}{c}\text { Confeito de cereal } \\
\text { com alfarroba }\end{array}$ & Produto A & Produto B & Produto C \\
\hline Valor Calórico & $135 \mathrm{Kcal}$ & $119 \mathrm{Kcal}$ & $148 \mathrm{Kcal}$ & $132 \mathrm{kcal}$ \\
\hline Carboidratos & $23 \mathrm{~g}$ & $18 \mathrm{~g}$ & $19 \mathrm{~g}$ & $19 \mathrm{~g}$ \\
\hline Proteínas & $1,9 \mathrm{~g}$ & $1,0 \mathrm{~g}$ & $1,7 \mathrm{~g}$ & $1,0 \mathrm{~g}$ \\
\hline Gorduras Totais & $4,1 \mathrm{~g}$ & $4,9 \mathrm{~g}$ & $7,2 \mathrm{~g}$ & $4,8 \mathrm{~g}$ \\
\hline Gorduras Saturadas & $1,1 \mathrm{~g}$ & $2,9 \mathrm{~g}$ & $3,9 \mathrm{~g}$ & $1,8 \mathrm{~g}$ \\
\hline Fibra Alimentar & $1,6 \mathrm{~g}$ & $0,7 \mathrm{~g}$ & $1,2 \mathrm{~g}$ & $1,2 \mathrm{~g}$ \\
\hline Sódio & $2,8 \mathrm{mg}$ & $37 \mathrm{mg}$ & $72 \mathrm{mg}$ & $36 \mathrm{mg}$ \\
\hline
\end{tabular}

Não foram observadas diferenças significativas no que diz respeito ao valor calórico e teor de carboidratos. Todavia, destacam-se os teores de gordura saturada $(1,1 \mathrm{~g})$ e fibras alimentares $(1,6 \mathrm{~g})$ do confeito de alfarroba, em relação aos demais produtos.

O teor de gordura saturada apresentou redução de 38 a $72 \%$ em relação aos produtos similares. Diversos estudos relacionam o consumo elevado de gordura saturada com o acúmulo de gordura nas artérias, resultando na obstrução de vasos sanguíneos pela formação de placas de ateroma (MÁRQUEZ, 2001; LOTTENBERG, 2009; SANTOS, 2013).

Neste estudo a quantidade de fibra se destacou em relação aos produtos similares do mercado, com valores de 25 a 56\% superiores. Ressalta-se que a quantidade de 1,6g fibra, na porção de 30g, classifica o produto como fonte (BRASIL, 2012). O papel da fibra alimentar é notório na prevenção de doenças intestinais, como constipação e câncer de cólon, além de contribuir no controle do colesterol sanguíneo e glicemia pós-prandial (MARLETT; MCBURNEY; SLAVIN, 2002).

\section{CONCLUSÃO}

Obteve-se um confeito de aveia com cobertura de alfarroba isento de lactose e fonte de fibra alimentar com boa aceitação para todos os atributos sensoriais, com alto Índice de Aceitabilidade e intenção de compra. Desse modo, o confeito de aveia com alfarroba consiste em uma boa opção para indivíduos intolerantes à lactose e para aqueles que buscam um produto prático e saudável.

\section{REFERÊNCIAS}

$A B I A D$. O papel da indústria na Análise de Impacto Regulatório. 2011. Disponível em: <http://www.abiad.org.br/images/conteudo/estudos/OPapeldalndnaAIR27102011.pdf>. Acesso em: 01 set. 2012.

BOWER, J. A.; WHITTEN, R. Sensory characteristics and consumer liking for cereal bar snack foods. Journal Sensory Studies, v. 15, n. 3, p. 327-45. 2000. 
BRASIL. ANVISA - Agência Nacional de Vigilância Sanitária. Portaria oㅡ 27 de 13 de janeiro de 1998 - Regulamento Técnico referente a Alimentos para Fins Especiais. Diário Oficial da União. 13 jan. 1998.

técnico sobre informação nutricional complementar. Disponível em: <bvsms.saude.gov.br/bvs/saudelegis/anvisa/2012/rdc0054 1211 2012.html>. Acesso em: 18 mar. 2013.

FLORES, F. S. Projeto restaurante com cardápio livre de glúten e lactose. Porto Alegre: Universidade Federal do Rio Grande do Sul, 2010.

FRYE, R. E. Lactose intolerance. Boston: Clínica Fellow, Departamento de Neurologia, Hospital de Crianças de Boston, Escola Médica Harvard, 2002.

LOTTENBERG, A. M. P. Importância da gordura alimentar na prevenção e no controle de distúrbios metabólicos e da doença cardiovascular. Arquivos Brasileiros de Endocrinologia Metabólica, v. 53, n. 5, pp. 595-607, 2009.

MARLETT, J. A.; MCBURNEY, M. I.; SLAVIN, J. L. American Dietetic Association. Position of the American Dietetic Association: health implications of dietary fiber. Journal American Dietetic Association, v. 102, p. 993-1000, 2002.

MÁRQUEZ, L. R. A fibra terapêutica. 2. ed. São Paulo: Byk Química, 2001. p. 14.

MATTAR, R.; MAZO, D. F. C. Intolerância à lactose: mudança de paradigmas com a biologia molecular. Revista da Associação de Médica Brasileira, v. 56, n. 2, pp. 230-236, 2010.

MURPHY, P. Countlines and cereal bar. In: JACKSON, E. B. (ed.) Sugar confectionery manufacture. London: Blackie Academic \& Professional, 1995. p. 287-297.

ÖZCAN, M. M. et al. Some compositional properties and mineral contents of carob (Ceratonia siliqua) fruit, flour and syrup. International Journal Food Science Nutrition, v. 58, n. 8, p. 652658. 2007.

PALAZZOLO, G. Cereal bars: they are not just for breakfast anymore. Cereal Foods World, v. 48, n. 2, p. 70-2, 2003.

SANTOS, R. D. et al. I Diretriz sobre o consumo de gorduras e saúde cardiovascular. Arquivos Brasileiros de Cardiologia, v. 100, n.1, suppl. 3, pp. 1-40, 2013.

STONE, H.; SIDEL, J. L. Sensory evaluation practices. 2. ed. London: Academic Press, 1993.

TEIXEIRA, E.; MEINERT, E. M.; BARBETTA, P. A. Análise sensorial de alimentos. Florianópolis: UFSC, 1987.

UNICAMP. Tabela Brasileira de Composição de Alimentos - TACO. 4. ed. Campinas. São Paulo, 2011. 